\title{
Saber objetivo sobre la subjetividad en psiquiatría, desde la filosofía y el psicoanálisis.
}

Objective knowledge about subjectivity in psychiatry, from philosophy and psychoanalysis.

Héctor Hueso Holgado a , Fanny Cuervo Díaz ${ }^{\text {. }}$

${ }^{a}$ Psiquiatra de la Unidad de Salud Mental de la Vera-Icod (Hospital Universitario Nuestra Señora de la Candelaria de Tenerife, España) y Psicoanalista Asociado de la Asociación Venezolana de Psicoanálisis: IPA y FEPAL. ${ }^{b}$ Pediatra. Médico del Servicio Urgencias Pediátricas Dr. Jaime Cháves Hernández. Santa Cruz de Tenerife, España.

Correspondencia: Héctor Hueso Holgado (hhuesoh@hotmail.com)

Recibido: 06/07/2012; aceptado con modificaciones: 06/01/2013

RESUMEN: Se propone que para no perder la identidad de la psiquiatría debe mantenerse la interdisciplinariedad entre los distintos modos de acceder a lo mental y al sentido la existencia, más allá de lo biológico. Intentando un "saber objetivo sobre la subjetividad", ya que la ciencia actual no aborda adecuadamente aspectos relativos a la existencia, al sujeto, al pensamiento no científico, ni mucho de lo que constituye el funcionamiento psíquico. En este sentido, también se hará referencia a la reciente investigación neurocientífica sobre el apego en la infancia, que apoya la constitución temprana de las bases neurológicas de los patrones de relación intersubjetiva que luego caracterizan al individuo.

PALABRAS CLAVE: psicoanálisis, psicoterapia, filosofía, apego, subjetivo.
ABSTRACT: It is proposed that to keep the identity of psychiatry should be kept interdisciplinarity between different ways of access to the mental and to the sense of the existence, beyond the biological. Trying an "objective knowledge about subjectivity", since modern science does not adequately address current issues relating to the existence, the subject, the non-scientific thinking, and not much of what constitutes mental functioning. In this regard, reference will be made to recent neuroscience research on attachment in infancy, which supports the early establishment of the neural basis of intersubjective patterns that subsequently characterize the individual.

KEY WORDS: psychoanalysis, psychotherapy, philosophy, attachment, subjective.

Agradecimientos:

A la realización de éste articulo han contribuido los estimulantes aportes, "interdisciplinarios" por demás, del resto de los participantes de la mesa redonda en que fue presentado: Juan de la Fuente Portero (psiquiatra), Laura Alarcó Ubach (psicóloga) y Amaia Vispe Astola (enfermera). A ellos nuestro agradecimiento.

Introducción y contexto filosófico al inicio del siglo XX.

El título de la mesa redonda en la que ha sido presentado este trabajo, "Interdisciplinariedad e intersubjetividad en el contexto institucional", sirve de marco al mismo. Se propone que para no perder la identidad de la psiquiatría debe mantenerse la interdisciplinariedad entre los distintos modos de acceder a lo mental y al sentido la existencia, más allá de lo biológico. Intentando, como dijo André Green, psicoanalista francés fallecido en enero de este año, un "saber objetivo sobre la subjetividad" (1), ya que coincidimos con en él en que la ciencia actual no aborda adecuadamente aspectos relativos a la existencia, al sujeto, al pensamiento no cien- 
tífico, ni mucho de lo que constituye el funcionamiento psíquico (tema sobre el que se centró un artículo anterior y al cual éste complementa (2)). En este sentido es interesante observar que al buscar las palabras claves para el resumen en el Medical Subject Headings (MeSH) del índex Medicus/Medline de la National Library of Medicine de Estados Unidos, no se encontraron registradas palabras como: intersubjetivo; objetividad; subjetividad; perspectivismo; hermenéutica; constructivismo, así como tampoco su traducción al inglés, lo cual evidencia la escasa cabida de estos conceptos en el ámbito médico, quedando relegado al de las humanidades y la filosofía. Dirijamos entonces primero la visión hacia la filosofía.

\section{Contexto filosófico al inicio del siglo $X X$.}

Paul Boghossian (3), catedrático de filosofía de la Universidad de New York, ha descrito como a través de la historia de la filosofía se ha ido alternando pendularmente, y con distintos nombres, entre el objetivismo y el subjetivismo. Aclaramos que no nos referimos a acepciones más recientes de objetivismo, como por ejemplo, el de Ayn Rand, que relaciona la búsqueda de felicidad con el sistema capitalista, sino con el objetivismo como fue planteado originalmente en la antigua Grecia. En tal sentido, simplificando mucho, el objetivismo, también llamado dogmatismo, realismo o filosofía de la trascendencia considera que es el "objeto" el que determina lo percibido y se propone conocer las "cosas en sí", es decir, en su trascendencia, por tanto, la verdad tiene que ser una para todos y en todo lugar y tiempo. Entre los iniciadores del objetivismo están Aristóteles, que se oponía al idealismo de su maestro Platón, y Santo Tomás de Aquino. Aun así, Hirschberger (4) ha destacado que para Aristóteles la física (ciencia de la naturaleza en general) se ocupa sólo de un caso especial del ser: el que se manifiesta a los sentidos; mientras la metafísica se ocupa de ese ser más profundo, que precede al otro y se da a conocer en la manifestación. Por lo cual, desde entonces se enfrentan el idealismo y el realismo con sus afirmaciones de lo que es verdadero ser.

Por el contrario, el subjetivismo o idealismo considera que las apariencias de las cosas dependen del sujeto que las observa y, por tanto, de sus ideas, prejuicios e idiosincrasia, de modo que no existiría una verdad universal (3). Entre ellos, aunque con notorias diferencias, comparten postulados subjetivistas Platón, San Agustín, Hume, Kant, Hegel, Nietzsche y más recientemente Wittgenstein, Thomas Kuhn, Richard Rorty e Hilary Putnam, entre otros. Sin embargo, según Lakoff y Jonhson (5), lingüista y filósofo respectivamente, no es cierto que haya que elegir entre objetivismo y subjetivismo, ya que hay situaciones de vida donde corresponde ser objetivo y otros donde corresponde ser subjetivo. La subjetividad comprende aspectos como la estética, la moral, la conciencia espiritual, el arte y la 
ORIGINALES Y REVISIONES

poesía, los sentimientos e intuiciones, las metáforas (y con ellas las sutilezas del lenguaje) y los mitos, presentes en todas las culturas.

Pasando al siglo XX, el positivismo (una de las formas del objetivismo que imperó desde mediados del siglo anterior y se fue disolviendo entre las dos Guerras Mundiales) reivindicaba a la ciencia como garantía de un progreso indetenible y al conocimiento científico como el único válido, y postulaba que la ciencia sólo estaba constituida por aseveraciones empíricamente verificables, aunque hoy en día el verificacionismo ha sido abandonado incluso por la física (6).

Tanto Freud como algún tiempo después Karl Popper estuvieron influenciados por el positivismo, a pesar de haberle hecho serias críticas. Popper negó que el psicoanálisis fuese una ciencia natural ya que no era falsable, sin embargo, si lo consideraba una "interesante metafísica psicológica" y le otorgaba, como a otras ideas metafísicas, cierto grado de verdad (7). Aun siendo un objetivista, no sólo no estaba en contra de la metafísica, sino que sostuvo que algunas de ellas han constituido programas de investigación y se han transformado (como en el caso del atomismo antiguo) en teorías controlables; que desde el punto de vista psicológico la investigación se vuelve imposible sin la fe en ideas de naturaleza metafísica; y que las metafísicas son criticables, aunque no sean falsables (8).

Posteriormente, sus propios discípulos y otros epistemólogos, como Kuhn, Feyerabend, Lakatos y Habermas, también cuestionaron la visión positivista estrecha de la ciencia. Habermas, uno de los filósofos de la Escuela de Frankfurt, coincidía con Popper en criticar a Freud por considerar al psicoanálisis como una ciencia natural, sin embargo, si lo consideraba una forma privilegiada de conocimiento ya que se oponía al monismo metodológico de Popper, pues cada tipo de conocimiento tiene su especificidad y la ciencia natural es sólo un tipo de conocimiento. Por su parte, Lakatos (también objetivista, pero crítico con algunas concepciones popperianas) señaló que el psicoanálisis no anticipa nuevos hechos y tampoco cumple el criterio de refutabilidad, pero que tampoco lo hace la dinámica ni la Teoría de la gravitación de Newton (9).

Si bien los positivistas lógicos o neopositivistas del Círculo de Viena y de Berlín consideraron que los postulados metafísicos carecían de sentido, los filósofos analíticos británicos les objetaron que, además de funciones morales, políticas y religiosas, "lo que comienza como metafísica puede acabar siendo ciencia". Otros filósofos analíticos posteriores, como John Watkins y Joseph Agassi, han defendido la utilidad científica de la metafísica y señalan que "las ideas metafísicas pertenecen a la investigación científica en cuanto ideas reguladoras de una importancia decisiva". Feyerabend dice que "para ser buenos empiristas es preciso producir diversas teorías metafísicas, para recuperar hechos que puedan contradecir a una teoría imperante (hechos que de otro modo sería imposible hallar) para maximizar el contenido de una teoría" (8).

Actualmente muchos psicoanalistas concuerdan en que el psicoanálisis no es una ciencia natural, pero no por ello deja de ser empírica y observacional (como 
observacional y no experimental fue la investigación de Darwin) pues trabaja con una teoría pragmática de la verdad. Como dijo Green, es una ciencia del sujeto. Sujeto que se convirtió en centro de la discusión filosófica en el siglo XX.

\section{Del sujeto a la intersubjetividad de los filosofos del diálogo}

La destructividad de las dos guerras mundiales, el nihilismo ideológico y los genocidios, confrontaron al optimismo positivista reinante y los filósofos se interrogaron sobre el poder, el marxismo, el fascismo y el nazismo. Entre ellos, los existencialistas se plantearon el sentido de la vida y Sartre defendió la ineludible libertad de elegir la existencia.

Oponiéndose a ellos, surgió el movimiento heterogéneo de los estructuralistas, quienes consideraban que lo fundamental no era el sujeto, sino las estructuras inconscientes comunes a los seres humanos de distintas culturas, especialmente la estructura lingüística (10). Pero al estructuralismo se le reprochó ser un antihumanismo que acababa con el sentido de lo vivido por el sujeto. Así, con el mayo francés del 68, comenzó su decadencia y tomaron el relevo los llamados posestructuralistas o postmodernos, como Derrida, que llegaron al escepticismo radical al considerar a la verdad inalcanzable, porque las interpretaciones son ilimitadas. Después se volverá a ello.

Por otra parte, las mencionadas circunstancias condujeron a los así llamados filósofos del diálogo y la intersubjetividad, a descentrar al sujeto (en lo cual se asemejan a los estructuralistas) rescatando el valor del otro, de lo intersubjetivo y del diálogo. Entre estos están: Heidegger, Buber, Wittgenstein, Merleau-Ponty, Lévinas y Gadamer; y comparten haber vivido las dos guerras mundiales en las que casi todos combatieron, fueron prisioneros y/o sufrieron pérdidas personales, lo que influyó en que también compartieran el dar la primacía al otro sobre el sujeto, al plantear que el "yo" se constituye a partir de un "tú" y un "nosotros". Además, se opusieron al dualismo mente-cuerpo y sujeto-objeto y, en cambio, proponían la "intersubjetividad".

En atención al espacio sólo podemos esbozar muy sucintamente algunas de sus ideas con la expectativa de incentivar al lector a profundizar en su conocimiento (recomendamos la síntesis que hizo Donna Orange en su libro Thinking for Clinicians (11)). Resumiendo mucho, estos comenzaron por seguir los lineamientos de su maestro y/o modelo, Martin Heidegger, cuyo existencialismo difería del de Sartre al subrayar que éste sobreestimaba la autonomía y atribuía un exagerado sentimiento de libertad. Consideraba que el humanismos antropocéntrico niega que siempre estamos en el mundo; que somos simplemente ser-en-el-mundo (Dasein); que no existe un sujeto sin mundo, ni un yo aislado sin los otros, sino que es una 
ORIGINALES Y REVISIONES

relación -más que un cerebro- la que constituye a la conciencia. Además, el hombre vive en la casa del lenguaje, el cual es la casa del ser (12).

Así, Martin Buber señala que el Yo-Tú antecede al Yo, el sujeto-Tú es indispensable para que aparezca el sujeto-Yo. En otras palabras, acentúa mucho más la relación sujeto-objeto que la consideración del sujeto, como hacen otros. Comprometía al clínico a encontrase con el otro como otro ser humano en un íntimo Tú, como un compañero de diálogo y no como una instancia de cualquier categoría. Su obra ha servido de apoyo a los psicoterapeutas humanísticos, especialmente a Carl Rogers en los años cincuenta y más recientemente por la terapia gestáltica relacional contemporánea (13).

Maurice Merleau-Ponty, se oponía a todo dualismo (mente-cuerpo; dentrofuera; sujeto-objeto) e intentó reemplazar el cogito cartesiano (pensamiento) por una subjetividad entrelazada con los otros. Para él no existe un ser interno privado, sino sólo el "ser encarnado y perceptivo en el mundo" (14). Es interesante observar la similitud entre el concepto filosófico de Merleau-Ponty sobre la "intersubjetividad encarnada (o incorporada)" y el concepto de "embodied simulation" ("simulación incorporada" o "simulación encarnada") que plantean Gallese y colaboradores, apoyándose en los descubrimientos sobre las "neuronas en espejo" (15). Merleau-Ponty (que se relacionó mucho con el existencialismo, la fenomenología, la psicología experimental y la biología) consideró que la Gestalt, la fenomenología de Husserl y el psicoanálisis ofrecían algo indispensable para entender la vida humana, pero perdían la perspectiva de la "intersubjetividad encarnada". Sus ideas básicas constituyen tanto un acercamiento como un desafío a Freud, Marx y Sartre. Lo que llevó a Lacan a intentar inicialmente aliarse con él aunque luego, no estando satisfecho con su postura y necesitando un basamento filosófico, invitó a Paul Ricoeur a sus seminario, pero éste finalmente le dijo que no podía entender sus discursos y rompieron la relación.

Ludwig Wittgenstein también pensaba que la dialéctica mente-cuerpo es un falso problema porque se intenta reducir una forma de descripción a otra y porque se emplea el vocabulario mental en el contexto equivocado, por ello consideró que debería aceptarse que los seres humanos pueden describirse de formas diversas, por ejemplo, en términos mentales o biológicos. Su defensa del principio de verificación en su gran obra, Tractatus lógico-philophicus, fue interpretada por los miembros del Círculo de Viena como si fuera la Biblia del neopositivismo. Sin embargo, Wittgenstein, aunque quería comprender el funcionamiento del lenguaje de la ciencia, a diferencia de los neopositivistas, le interesaba lo que la ciencia no puede decir: "Sentimos que aun cuando todas las posibles cuestiones científicas hayan recibido respuesta, nuestros problemas vitales aun no se han rozado en lo más mínimo. La ciencia calla sobre lo que nos es más importante: la ética y la religión" (16). Además, especialmente la segunda parte de su obra cuando se alejó de la filosofía analítica de su maestro Bertrand Russell, se orientó hacia el "otro", 
la ética y la religión, como hicieron, sobre todo, Buber y Emmanuel Lévinas. Éste último tampoco creía que lo más importante fuera el ser, sino la primacía del otro, por tanto, defendió el restablecimiento de la ética como la primera filosofía. Lévinas, habiendo sufrido un campo de concentración, en los que perdido a casi toda su familia, se decepcionó de su maestro Heidegger y proclamó que la cara del sufrimiento del otro coloca una infinita e irrealizable demanda en quien la mira.

Finalmente, "el gran viejo", el último en morir en 2002 a los 102 años, HansGeorg Gadamer, definió su hermenéutica diciendo que en lugar de la primacía de la subjetividad y autoconciencia se sitúa el otro. Su pasión fue la práctica y el proceso de la conversación, y su hermenéutica el proceso de entendimiento del diálogo. Diálogo que se basa en la defensa de la propia postura pero con la admisión fundamental de que el otro podría tener razón (17). Así, rechazó las formas autoritarias de comunicación (aun reconociendo la legítima autoridad de la tradición) y se opuso a la pretensión de la interpretación del experto, descartando la noción de la empatía como forma de leer la mente del autor o del paciente. Defendió por tanto, una hermenéutica de la confianza, como opuesta a la hermenéutica de la desconfianza que Ricoeur le atribuía a Nietzsche, a Freud y a Marx.

Más adelante haremos alguna otra referencia a estos filósofos del diálogo y lo intersubjetivo, pero ahora hay que señalar que diversos hallazgos de las neurociencias parecen sustentar sus postulados. Así, desde la vertiente experimental, tanto Ammaniti y Trentini (18) como Gallese (15) (colaborador del descubrimiento de las "neuronas espejo") aportan una perspectiva neurobiológica sobre la intersubjetividad, según la cual las instancias del sí mismo y del "espacio nosotros" tienen como sustrato circuitos neuronales. Gallese considera que este basamento neurológico apoya a la intersubjetividad de la filosofía fenomenológica.

Sin embargo, desde el punto de vista de la "filosofía de la mente", coincidimos con Wittgenstein y el filósofo de la ciencia norteamericano, Hilary Putnam (19) en el peligro de intentar reducir la forma de descripción de lo mental a lo cerebral y creemos que probablemente deba aceptarse que son ámbitos que requieren metodologías diferentes y que alcanzan saberes distintos. Incluso los que creen que un estado mental es sólo un estado cerebral, ya no piensan que estén originados en un área específica o tenga los mismos sustratos. Por ejemplo, Joaquim Fuster (20), neurocientífico español, dice que la mente funciona como un código relacional similar al lenguaje y, por tanto, pretender estudiar las partes de la mente sería como intentar entender el significado de lo que dice una carta estudiando la composición química de la tinta.

Tanto los hallazgos de las neurociencias como los de los filósofos de la intersubjetividad, han influido en el psicoanálisis anglosajón, imponiéndose la llamada "two-person psychology" o lo que algunos llaman un "giro intersubjetivo en psicoanálisis" (21). 
ORIGINALES Y REVISIONES

\section{Psicoanálisis intersubjetivo y perspectivismo}

El perspectivismo apareció como teoría alternativa al objetivismo y al subjetivismo, afirmando que la realidad puede ser apreciada desde diferentes vértices. El perspectivismo de Nietzsche estaba relacionado a la subjetividad del observador, en cambio el de Ortega y Gasset dependía más del objeto observado y lo describió usando la metáfora de la sierra del Guadarrama, que es distinta según se la mire desde Madrid o desde Segovia (22).

También en psicoanálisis, a partir de 1970, utilizando tesis hermenéuticas, postmodernas y socio-constructivistas, se planteó que el sentido del material psicoanalítico era construido (no reconstruido) entre las perspectivas del analista y analizado. Por ejemplo, Roy Schafer, apoyándose en Ricoeur y en Habermas, acentuó el carácter subjetivo del conocimiento en psicoanálisis y la imposibilidad del intérprete de ser un observador neutro (23). Schafer, Spence y Viderman han restado importancia a la "verdad histórica", destacando más bien la construcción de una "verdad narrativa".

Como Shafer y otros relacionistas, los intersubjetivistas norteamericanos, entre ellos Mitchel, Orange, Stolorow, Atwood, Beebe, Lachmann y Fosshage, han defendido el perspectivismo, pues asumen que hay que reconocer que sus teorías influyen en la captación del mundo subjetivo de sus pacientes y en la co-determinación del proceso analítico. Sin embargo, insisten en que sus teorías no plantean abstenerse de utilizar teorías que guíen y ordenen los datos clínicos (24).

En general, los psicoanalistas relacionistas e intersubjetivistas tienden a dar preeminencia a lo intersubjetivo sobre lo intrapsíquico (25) y consideran que son las relaciones y los afectos (más que las pulsiones) los que evolutivamente dan lugar a principios ("patrones temáticos o estructuras de significado") que inconscientemente organizan las experiencias emocionales y relacionales (26), por tanto focalizan en las rupturas de comunicación y en los fallos de la empatía. Tienen el mérito de haber contribuido a ampliar la concepción de los fenómenos de transferencia y contratransferencia, pero comparto las críticas de Gabbard (27), Kernberg (28), Deprati (29) y Bleichmar (30) al señalar que a veces representan una hermenéutica extrema que subestima el poder de la transferencia y de todo aquello que preexiste en el paciente y que éste despliega, incluso independientemente de lo que haga el terapeuta. Así como subestiman el componente biológico, intrapsíquico y pulsional -sin entrar aquí en cuánto de lo pulsional es biológico, adquirido o ambiental (31). 


\section{La limitación hermenéutica para captar el psiquismo}

Entendiendo hermenéutica, de manera general, como la interpretación de textos tanto escritos como verbales, algunos defienden planteamientos similares a los de Wittgenstein cuando dijo que "no puede haber experiencia subjetiva previa al lenguaje" (32). Entre los que piensan que lo psíquico sólo está representado por el lenguaje, en nuestra opinión, hay quienes llegan a una simplificación extrema e ingenua y, evitando la hermenéutica de la sospecha (como decía Ricoeur), pasan a lo que a nuestro parecer es la hermenéutica ingenua que piensa que sólo es "verdad" lo manifiesto y nada se esconde detrás. Por tanto, consideramos, que los aportes hermenéutico y narrativo son insuficientes para la captación de la intersubjetividad y del psiquismo cuando se restringen a la traducción del lenguaje hablado y no incluyen el discurso implícito en lo no dicho y el de los lenguajes inconsciente, preverbal, afectivo, artístico y gestual (33). De hecho, como antes vimos, la investigación reciente sobre la memoria implícita y las neuronas espejo, entre otras, apoya que el psiquismo comienza a desarrollarse a través de intercambios intersubjetivos no verbales y esto también puede verse en los distintos modos de comunicación que mantenemos con algunos animales o en cómo estos se comunican sus estados emocionales entre sí (por ejemplo, los estudios con chimpancés muestran que pueden distinguir al menos diez expresiones faciales (34).

El aporte fundamental de la metapsicología freudiana, como su nombre lo indica, es que lo psíquico va más allá de la consciencia, razón por la cual psicoanalistas de diversas tendencias se oponen a considerarlo una hermenéutica, dadas las limitaciones de ésta última $(1,35,36)$. De hecho, si lo hermenéutico se reduce a la consciencia se pierden los aportes, no sólo del psicoanálisis, sino de la reciente investigación neurocientífica y de la teoría del apego, la cual apunta hacia que los patrones interactivos entre los infantes y sus cuidadores, crean cambios duraderos en las redes cerebrales y conducen a estilos de apego y patrones afectivos, que se forman antes del desarrollo de la memoria explícita (o sea, de forma implícita y presimbólica) y tienden a permanecer durante la vida $(37,38)$.

En tal sentido, el común denominador de tres de los más importantes investigadores de la temprana infancia: Meltzoff, Trevarthen, y Stern, es señalar que las formas simbólicas de comunicación están construidas sobre formas presimbólicas y tienden a ser implícitas (39). Por ejemplo, los experimentos de Meltzoff y los de Trevarthen documentan que desde sus primeras horas el bebé y sus cuidadores se comunican de forma "inmediata, irracional, no verbalizada y ateórica", y que son formas representacionales rudimentarias, innatas, quizás basadas en las "neuronas espejo". Probablemente en parte innatas y en parte adquiridas. Sin embargo, la psicoanalista interpersonalista Beebe y sus colaboradores (39) opinan que estos tres autores desatienden el hecho de que lo que caracteriza la interacción madre hijo son 
ORIGINALES Y REVISIONES

tanto las similitudes como las diferencias, así como que los miembros de la pareja se influencian mutuamente y los patrones son co-construidos. Además, dicen, omiten la propia auto-regulación del bebé, incluso a nivel fisiológico.

Algunos psicoterapeutas de técnica narrativa, al igual que los psicoanalistas intersubjetivos, se apoyan en tesis postmodernistas y constructivistas, pero a diferencia de estos últimos plantean que no hay significados ocultos a encontrar tras el relato de los pacientes y, por tanto, el terapeuta es un experto conversacional que no intenta que lo dicho por el paciente encaje en esquemas, como podrían ser los conductistas, cognitivistas, psicoanalistas, estructuralistas, sistémicos, etc. (40). Sin embargo, no es posible prescindir de esquemas referenciales y metodológicos, tal como destacó Heidegger al señalar que "quien niega su ubicación hermenéutica corre el peligro de exponerse aún más acríticamente a ella" (41).

Hay que recordar que al subjetivismo extremo del posmodernismo y deconstructivismo francés se opusieron filósofos alemanes, como Heidegger, Gadamer y Habermas, que no estaban de acuerdo en que cualquier interpretación o cualquier narrativa tuviesen igual valor. En palabras de Mitchell, de cuyo grupo surgió el psicoanálisis relacional, hay muchas maneras de pintar un jarro con flores pero ello no significa que todas valgan igual (42).

Si bien Gadamer y Heidegger consideraban que al no existir una verdad absoluta, última y atemporal, sería imposible alcanzar una metodología hermenéutica sistematizada y con reglas generales para lograr el estatus de ciencia del espíritu (tal como habían intentado infructuosamente desde el siglo XIX, Schleiermacher, Droysen, Dilthey y Betti) aun así, si propusieron un método: reconocer los propios prejuicios y la propia subjetividad; la propia ubicación hermenéutica del intérprete, para poder contrastarla con el texto del otro [del paciente]. Heidegger dijo: "Lo que importa no es que se cierren los ojos ante este «malvado» círculo deseando su desaparición para lograr cualquier objetividad inexistente. Lo decisivo es no salirse de este círculo, sino introducirse en él de la manera correcta" (43).

Por otra parte, Gadamer concordó con su maestro Heidegger en que las ciencias del espíritu, tal como propuso Helmholtz (el científico de la naturaleza), tienen más que ver con el uso de un «tacto psicológico... una inducción artística... una sensibilidad» (43).

Esto es similar a lo señalado por el director de la Scala de Milán, Riccardo Muti (44), al decir que la función del director es tomar los sentimientos de las almas de los músicos, no marcar los tiempos ni las notas (que son sólo la expresión concreta de los sentimientos), puesto que señalar las notas es algo que cualquiera podría hacer y, en cambio, "dirigir es la profesión más difícil del mundo". Los comentarios de éste músico encajan muy bien con lo que ha sostenido Daniel Stern sobre lo que llama "sintonía afectiva", que es un emparejamiento de formas de sen- 
timiento, más que de palabras o de conductas, y que considera un criterio central de su teoría de la comunicación entre infante y madre (39).

Es que los "movimientos" del director de orquesta y los del terapeuta, no sólo tienen que ver con las notas y las palabras. Tienen también que ver con eso que se ha llamado "feeling", más relacionado con la llamada memoria no verbal o implícita, o sea, con aspectos de la terapia que no producen cambios por medio de la palabra (la interpretación mutativa), la memoria declarativa y los simbolismos, sino por intercambios intersubjetivos que Stern y sus colaboradores del "Process of Change Study Group" en Boston (45) han llamado "moments of meeting" (momentos de encuentro) en los que el encuentro produce sucesos mutativos; un "algo más" que no se registra simbólicamente y que por tanto son de difícil traducción en términos hermenéuticos.

Además, cada vez se está dando más importancia en psicoanálisis a los enactments (que puede ser traducido como "puesta en acto" o "en escena") que son patrones relacionales y emocionales codificados neurológicamente de modo implícito en las relaciones tempranas (según algunos estudios: a nivel de amígdala, sistema límbico y hemisferio derecho), y que se activan automática e inconscientemente dentro de la relación intersubjetiva. Al ser detectadas estas puestas en acto y llevadas a concientización verbal, se crea la oportunidad para la integración emocional y neurológica (37).

\section{Conclusiones}

Se ha señalado que hay ámbitos correspondientes al subjetivismo y otros al objetivismo y que la suma de perspectivas puede aportar visiones complementarias, como decía Ortega sobre la sierra del Guadarrama. Así, la complejidad del psiquismo es una Gestalt cuya totalidad es más que la suma de sus partes y, por tanto, no es posible pretender un objetivismo ingenuo que aspire a alcanzar la verdad de la "cosa en sî" (como ya lo habían descartado empiristas como Hume, o idealistas como Kant). Sólo renunciando a esta pretensión podemos creer que es posible, utilizando la expresión de Green, alcanzar un "saber objetivo sobre la subjetividad". De tal manera que el error del objetivismo sería considerar al objeto-mente del paciente el único responsable del conocimiento (que es el reproche que los psicoanalistas intersubjetivistas hacen a Freud), mientras el error del subjetivismo sería excederse en lo que el sujeto-terapeuta impone al mismo (que es el reproche que se les hace a los intersubjetivistas).

En psiquiatría, el tan repetido paradigma bio-psico-social tiende a convertirse más en BIO que en psico-social pero, a nuestro modo de ver, debemos rescatar el psico, con PSI de psiquiatra, de psicólogo, de psicoterapeuta, pues pareciera que 
ORIGINALES Y REVISIONES

hay una psiquiatría que aspira a convertirse en neurología o neurociencias, aunque ya estas tienen su lugar. Por el contrario, consideramos que lo que debemos preservar es el espacio dentro de la Medicina y la ciencia para lo psíquico, lo subjetivo, lo relacional, lo afectivo. No es cuestión de despreciar a las neurociencias, sino de servirles de orientadores de lo mental como lo propuso el premio nobel Eric Kandel $(46,47)$. Hablamos de preservar al ámbito psíquico, al subjetivismo y al "gris" de la "lógica borrosa" (al que hemos dedicado otro artículo (48)) en un punto intermedio entre el extremo del cientificismo, que privilegia lo racional-científico, y el otro extremo del relativismo deconstructivo. Con ese objetivo se han destacado las ideas de algunos filósofos del siglo XX que han rescatado la importancia del otro, del diálogo y el valor de la relación y el amor. Sin embargo, creemos que un énfasis exagerado en la consideración del otro, en perjuicio del Ser que proponía Sartre, tiene el peligro de restar responsabilidad al paciente sobre su propia vida. Conviene recordar lo que planteaba Heidegger en cuanto a que el "estar-con-los-otros", la "solicitud" o el "preocuparse por", más que evitar al otro su propio cuidado, debería consistir en ayudarles a conquistar la libertad de asumir su propio cuidado (12).

Postulados como los de los filósofos del diálogo podrían aportar ideas valiosas a los profesionales de la salud mental, pero en no pocas ocasiones la realidad institucional hace difícil su aplicación, dada la frecuente sobrecarga del facultativo, junto a la expectativa de resultados inmediatos por parte de pacientes y familiares. Lo que a veces conduce al desbordamiento de la capacidad receptora y a que la verdadera interdisciplinariedad en salud mental se vuelva más teórica que practicable.

Ahora, volviendo a lo subjetivo, no creemos que las ideas de los filósofos y psicoanalistas citados justifiquen cierta tendencia a ver a la psicoterapia como una especie de relación amigable, casi amorosa, donde "todo vale". No se trata de una acción meramente caritativa y "simpática". Por ejemplo, pese a que Martin Buber, defendió que la relación es recíproca, señaló que la relación de ayuda es asimétrica por naturaleza y nunca podrá desplegarse en una completa mutualidad (13). Igualmente, Merleau-Ponty describió la intersubjetividad como dos manos tocándose al estrecharse entre sí y, aunque la intersubjetividad siempre ha significado reversibilidad, concluye que hay una doble "asimetría": los otros me ven y me sienten como yo posiblemente no puedo sentirme, y yo me siento a mí mismo como los otros probablemente no pueden sentirme (14).

Considerar que la empatía, sólo se refiere a las partes "buenas" de las personas es olvidar que también hay que poder empatizar con lo más negativo que el paciente reprime en sí mismo y muchas veces proyecta en los demás, Hay que reconocer que existe la maldad (tan sólo hace falta abrir un periódico o mirar la historia) y que esta también tiene lugar en la narrativa. Si bien podemos cuestionar la "verdad" de la historia y admitir la relatividad de lo escrito, no se puede llegar a cuestionar que existiesen y aun existen holocaustos, como tampoco podemos re- 
escribir una historia personal excluyente de lo negativo y de las verdades dolorosas o vergonzosas.

Al igual que muchos hermeneutas no aceptan el "todo vale", no "todo vale si ayuda al paciente", pues no sólo habría que definir qué y cómo es lo que vale para el paciente, sino quién lo determina y cómo puede estructurarse una teoría de un modelo terapéutico si se va cambiando aleatoria e intuitivamente, momento a momento, lo que se cree que ayuda al paciente. Además, la postura del "todo vale" es peligrosamente omnipotente y otorga un poder de decisión omnisapiente al terapeuta, con la paradoja de que parece liberarlo de la responsabilidad de buscar arduamente el saber y del estudio de teoría y técnica psicoterapéutica, conformándose con su supuesto carisma o su supuesta buena, sensible o caritativa intencionalidad, pero careciendo de la sujeción y protección que brinda una metodología.

En este sentido, no creemos que la metodología psicoanalítica sea del todo una hermenéutica. Por el contrario, consideramos insuficiente el aporte hermenéutico si no incluye los otros modos de comunicación mencionados. Y aunque nuestra metodología sea inevitablemente dependiente del marco teórico del investigador, no por ello hay que creer que toda interpretación es tan válida como cualquier otra, ni hay que renunciar a alcanzar cierto grado de saber objetivo de la subjetividad.

"Lo que la psiquiatría le debe al psicoanálisis, es el haber recuperado, gracias a Freud, el sentido de su propia existencia [...] el del hombre vuelto a su naturaleza imaginaria” (Henri Ey (49)).

BIBLIOGRAFÍA:

(1) Green, A. Desconocimiento del inconsciente (Ciencia y psicoanálisis). En: Dorey, R., Castoriadis, C., Enriquez, E., Thom, R., Ménéchal, J., Fridman, et al. El inconsciente y la ciencia. Amorrortu Editores, 1991; p. 167-257.

(2) Hueso, H. Saber objetivo sobre la subjetividad, desde el psicoanálisis intersubjetivo y la filosofía del siglo XX. Videoconferencia en la Asociación Venezolana de Psicoanálisis (ASOVEP), 25-022012 .

(3) Boghossian, P. Introducción. En: El miedo al conocimiento. Contra el relativismo y el construccionismo. Alianza Editorial, 2009; p. 15-25.

(4) Hirschberger, J. Aristóteles, la idea en el mundo. En: Breve historia de la filosofía. Ed. Herder. 1964, 47-70.

(5) Lakoff, G y Johnson, M. Metaphors We Live By, University of Chicago Press, 1980, 185189. Recuperado Febrero 04, 2012 en: http://www.robertexto.com/archivo11/objetiv_subjetiv.htm

(6) Berrios, G. Editorial: Sobre la medicina basada en la evidencia. Psicoevidencias [revista electrónica]. 26-02-2010; [consultado 02-02-2012]: Disponible en:

http://www.psicoevidencias.es/Novedades/Editorial/editorial-sobre-la-medicina-basada-en-la-evidencia. $\mathrm{html}$ 
(7) Popper, K. El problema de la demarcación. En: Popper Escritos selectos. Compilador David Miller. México, Fondo de Cultura Económica, p. 131-142.

(8) Reale, G. y Antiseri, D. La epistemología postpopperiana. En: Historia del pensamiento filosófico y científico III Del romanticismo hasta hoy. Editorial Herder, 1988; p. 908-934.

(9) Clavel,F.Las críticas de Karl Popper al psicoanálisis. Signos Filosóficos, suplemento núm. 11, vol. VI, 2004, pp. 85-99. Recuperado Febrero 04, 2012 en: http://redalyc.uaemex.mx/pdf/343/34309906. pdf

(10) Reale, G. y Antiseri, D. El estructuralismo. En: Historia del pensamiento filosófico y científico III Del romanticismo hasta hoy. Editorial Herder, 1988; p. 824-840.

(11) Orange,D.Thinking for clinicians. Philosophical resources for contemporary psychoanalysis and the humanistic psychotherapies. Editorial Routledge, 2010.

(12) Heidegger, M. El estar-en-el-mundo como coestar y ser-sí-mismo. En: Ser y tiempo. Editorial Trotta, 2009; p. 134-149.

(13) Orange, D. Martin Buber. En: Thinking for clinicians. Philosophical resources for contemporary psychoanalysis and the humanistic psychotherapies. Editorial Routledge 2010; p. 15-34.

(14) Orange, D. Maurice Merleau-Ponty. En: Thinking for clinicians. Philosophical resources for contemporary psychoanalysis and the humanistic psychotherapies. Editorial Routledge. 2010; p. 70.

(15) Gallese, V., Eagle, M., y Migone, O. Intentional attunement: mirror neurones and neural underpinning of interpersonal relations. J. Amer. Psychoanal. Assn. 2007; 55:131-175.

(16) Reale, G. y Antiseri, D. Ludwig Wittgenstein, desde el <<Tractatus Logico-Philosophicus $>>$ hasta las <<Investigaciones filosóficas >>. En: Historia del pensamiento filosófico y científico III Del romanticismo hasta hoy. Editorial Herder, 1988; p. 581-591.

(17) Reale, G. y Antiseri, D. Hans-Gerg Gadamer y la teoría de la hermenéutica. En: Historia del pensamiento filosófico y científico III Del romanticismo hasta hoy. Editorial Herder, 1988; p. 555-566.

(18) Reis, B. Nosotros: Comentario sobre los trabajos de Trevarthen, Ammaniti y Trentini, y Gallese. Clínica e Investigación Relacional [revista electrónica] 2011; 5 (1): 98-117. [ISSN 1988-2939] [consultado 04-02-2012]: Disponible en: http://www.psicoterapiarelacional.es/CeIRREVISTAOnline/ Volumen51Febrero2011/tabid/761/Default.aspx

(19) Putnam, H. Wikipedia. [consultado 04-02-2012] Disponible en: http://es.wikipedia.org/ wiki/Hilary_Putnam

(20) Fuster, J. Entrevista de Eduard Punset a Joaquim Fuster. Palma de Mallorca, 24 de setiembre del 2011. Vídeo del programa: "El alma está en la red del cerebro" - emisión 110 (13/11/2011) - temporada 16. [consultado 04-02-2012] Disponible en: http://www.redesparalaciencia.com/6374/redes/ redes-110-el-alma-esta-en-la-red-del-cerebro

(21) Drozek, R. Intersubjectivity theory and the dilemma of intersubjective motivation. Psychoanalytic Dialoges, 2010; 20:540-560.

(22) Reale, G. y Antiseri, D. Los filósofos españoles. Ortega. En: Historia del pensamiento filosófico y científico III Del romanticismo hasta hoy. Editorial Herder, 1988; p. 942-945.

(23) Tessier,H.Las corrientes disidentes posteriores a la psicología del yo. «Les courants dissidents postérieurs à l'ego-psychology», extracto del libro de Hélène Tessier: La psychanalyse américaine, 2005; p. 61-78. Y en: Revista Alter, $N^{\circ} 6$ [revista electrónica] 2005 [consultado 04-02-2012] Disponible en: http://revistaalter.com/Revistas/Numero6/Las_corrientes_disidentes_posteriores_a_la_psicologia_del_ yo.htm 
(24) Stolorow, R. \& Atwood, G. Epílogo. En: Los Contextos del Ser: las Bases Intersubjetivas de la Vida Psíquica. Herder Editorial, 2004; p. 199-200.

(25) Stolorow, R. Introducción. En: World, affectivity, trauma. Heidegger and post-cartesian psychoanalysis. Rouletdge, 2011; p. 1-4.

(26) Stolorow, R. Psicoanálisis post- Cartesiano como contextualismo fenomenológico. En; World, affectivity, trauma. Heidegger and post-cartesian psychoanalysis. Rouletdge, 2011; p. 19-33.

(27) Gabbard, G. A Neurobiologically Informed Perspective on Psychotherapy. British Journal of Psychiatry 2000; 177:117-122.

(28) Nos, J. Entrevista a Otto Kernberg. Temas de Psicoanálisis. Revista de la Sociedad Española de Psicoanálisis [revista electrónica] [consultado 04-02-2012] Disponible en: http://www. temasdepsicoanalisis.org/entrevista-con-el-dr-otto-kernberg-2

(29) Deprati,D.Diferencias Entre Dos Corrientes Psicoanalíticas: La Interpersonal y la Relacional Reseña sobre: Are Interpersonal and Relational Psychoanalysis the same? Frankel, J.B. Contemp. Psychoanal. 1998; 34:485-500.Yen: Revista de Psicoanálisis [revistaelectrónica] julio 2000 Nº5 [consultado 04-02-2012] Disponible en: http://www.aperturas.org/articulos.php?id=0000124\&a=Diferencias-entredos-corrientes-psicoanaliticas-la-interpersonal-y-la-relacional

(30) Bleichmar, H. El Cambio Terapéutico a la Luz de los Conocimientos Actuales Sobre la Memoria y los Múltiples Procesamientos Inconscientes. Aperturas Psicoanalíticas. Revista de Psicoanálisis [revista electrónica] noviembre $2001 \mathrm{~N}^{\circ} 9$ [consultado 04-02-2012] Disponible en: http://www.aperturas. org/articulos.php?id=0000178\&a=El-cambio-terapeutico-a-la-luz-de-los-conocimientos-actuales-sobrela-memoria-y-los-multiples-procesamientos-inconscientes

(31) Green, A. The Intrapsychic and Intersubjective in Psychoanalysis. Psychoanal Q. 2000; 69: $1-39$.

(32) Mendoza, M. Nietzsche desde la hermenéutica analógica. En: Hermenéutica analógica, estética y cultura. Facultad de Filosofía y Letras, UNAM, México. 2008; p. 47-70.

(33) Taylor, C. The Ethics of Authenticity. Cambridge, MA: Harvard University Press. 1991; p. 34-35.

(34) Zawa, O. y Izumi, A. Planeta Tierra. Evolucionar para sobrevivir [consultado 07-04-2012] Disponible en: http://www.youtube.com/watch?v=c_VftYkcoA0

(35) Renik, O. Analytic Interaction: Conceptualizing Technique in Light of the Analyst's Irreducible Subjectivity. Psychoanal Q.1993; 62:553-571.

(36) Renik, O. The Analyst's Subjectivity and the Analyst's Objectivity. Int. J. Psycho-Anal., 1998; 79:487-497.

(37) Ginot, E. Intersubjectivity and Neuroscience: Understanding Enactments and Their Therapeutic Significance Within Emerging Paradigms. Psychoanal. Psychol. 2007; 24:317-332.

(38) Lyons-Ruth, K. Dissociation and the parent-infant dialogue: A longitudinal perspective from attachment research. J. Amer. Psychoanal. Assn. 2003; 51:883-911.

(39) Beebe, B., Rustin, J., Sorter, D. and Knoblauch, S. (2003). An Expanded View of Intersubjectivity in Infancy and its Application to Psychoanalysis. Psychoanalytic Dialogues 2003; 13: 805-841.

(40) Fernández L,A. y Rodríguez, B. La práctica de la psicoterapia: La construcción de narrativas terapéuticas. Ed. Descleé De Brouwer, 2002.

(41) Grondin, J. Heidegger: La hermenéutica como esclarecimiento de sí mismo del estarinterpretado existenciario. En: Introducción a la hermenéutica filosófica. Editorial Herder. 2002; p. 137 155. 
ORIGINALES Y REVISIONES

(42) Coderch, J. El espacio terapéutico y la autoridad del psicoanalista. En: La práctica de la psicoterapia psicoanalítica. El modelo interactivo en el campo del psicoanálisis. Editorial Ágora Relacional 2010; p. 141-158.

(43) Grondin, J. La hermenéutica universal de Gadamer. En: Introducción a la hermenéutica filosófica. Editorial Herder. 2002; p. 157-178.

(44) Muti, R. Discurso al recibir el reconocimiento como "Músico del Año", de América Musical [consultado 04-02-2012] Disponible en: http://www.youtube.com/watch?v=xhZct1H4bxI

(45) Stern, D.N., Sander, L.W., Nahum, J.P., Harrison, A.M., Lyons-Ruth, K., Morgan, A.C., et al. Non-Interpretive Mechanisms in Psychoanalytic Therapy: The 'Something More' Than Interpretation. Int. J. Psycho-Anal. 1998; 79:903-921.

(46) Kandel, E. A New Intellectual Framework for Psychiatry. Am J Psychiatry 1998; 155:457469.

(47) Kandel, E. Biology and the Future of Psychoanalysis: A New Intellectual Framework for Psychiatry Revisited. Am J Psychiatry 1999; 156,505-524.

(48) Hueso, H. Lógica borrosa y epistemología psiquiátrica. A propósito de la transexualidad. VITAE Academia Biomédica Digital. Facultad de Medicina. Universidad Central de Venezuela. [revista electrónica] Enero- Marzo, $2011 \mathrm{~N}^{\circ} 45$ [consultado 04-02-2012] Disponible en: http://vitae.ucv.ve/index_ pdf.php?module=articulo_pdf \&n=4303\&rv=9

(49) Roudinesco, E. La escuela freudiana de París: la reconquista. En: La batalla de los cien años. Historia del psicoanálisis en Francia (Tercera parte. Capítulo II). Editorial Fundamentos 1993; p. 101-164. 